XV.

Aus der chirurgischen Universitätsklinik zu Leipzig.

(Direktor: Geheimrat Prof. Dr. Trendelenburg.)

\title{
Ein Fall von Embolie der Lungenarterie nach der Methode von Trendelenburg operiert.
}

\author{
Von \\ Dr. med. Roderich Sievers, \\ Assistent der Klinik. \\ (Mit 1 Abbildung.)
}

Die Operation der Lungenarterienembolie, die Herr Geheimrat Trendelenburg zuerst auf der 79. Versammlung Deutsch. Naturf. und Ärzte (vgl. Zentralblatt f. Chir. 1907, Nr. 44) einem größeren Kreise bekannt gegeben, später nach wesentlicher Abänderung und Vervollkommnung der Technik in der Mediz. Gesellschaft zu Leipzig vom 28. I. 1908 (vgl. Zentralbl. f. Chir. 1908, Nr. 4) und neuerdings dem Chirurgenkongrel vorgeführt hat, hatte bisher keinen Erfolg am Menschen zu verzeichnen. Bei dem ersten Fall nach dem neuen Verfahren, über den Trendelenburg auf dem Kongreß berichtete, trat vor Beendigung der Operation der Tod ein.

Es wird daher der Bericht über die folgende zweite Operation von Interesse sein. Es ist mir dabei gelungen, sämtliche Emboli zu entfernen und die Operation glücklich zu beenden. Die zu Beginn der Operation pulslose Patientin erholte sich soweit, daß sie noch 15 Stunden lebte, erlag dann allerdings der zunehmenden Herzschwäche:

Die 48 jähr. Gutsbesitzersehefrau Bertha W. war am 16. April unter den Erscheinungen der Appendicitis (Schmerzen in der rechten Unterbauchgegend und einmaligem Erbrechen), akut erkrankt, hatte sich am 21. IV. ins Bett gelegt wegen Zunahme der Schmerzen und allgemeinem Übelbefinden. Seitdem bestand Verhaltung von Stuhl und Winden trotz Einläufen und Abführmitteln. Deswegen wies sie der Arzt am 24. IV. der Klinik zu. Die Frau gab an, außer ihren Unterschenkelgeschwüren sei sie stets gesund gewesen, habe 9 gesunde Kinder geboren, die alle am 
Ein Fall v. Embolie d. Lungenarterie n. d. Meth. von Trendelenburg oper. 283

Leben seien. Auf Befragen gab sie weiter an, niemals Gelbsucht, niemals Kolik- oder leichtere Schmerzanfälle irgendwelcher Art gehabt zu haben. Herz- oder Atembeschwerden hatten niemals bestanden.

Der Aufnahmebefund war folgender: sehr starke, etwas anämische, nicht ikterische Frau mit ängstlichem Gesichtsausdruck und frequenter, nicht kupierter Atmung. Temperatur 38,1 C in Axilla, Puls 108 von geringer Füllung und Spannung, regulär und äqual. Zunge bräunlichweiß belegt, wenig feucht. Kein Herpes labialis. Rachenorgane frei. Haut ohne Narben und Ödeme außer ausgebreiteten Ulcera cruris. Keine Anhaltspunkte für Venenthrombose.

Lungengrenzen stehen etwas hoch. Über der rechten Lunge hintenunten besteht etwa vier Querfinger breite Dämpfung, über der der Stimmtremitus nicht abgeschwächt ist. Im Bereich der Dämpfung hört man teilweise Bronchialatmen, teilweise vesikuläres Atmungsgeräusch von feinem Knisterrasseln begleitet. Über den übrigen Lungenpartien nichts Abnormes. Herzaktion regelmäBig, keine Geräusche, Dämpfung nicht vergrößert oder verschoben.

Der Leib ist stark anfgetrieben und gibt überall laut tympanitischen Schall. Durch die enorm fetten und gespannten Bauchdecken ist eine zuverlässige Palpation ausgeschlossen. Das rechte Hypochondrium zeigt mehr Empfindlichkeit auf Druck als die Gegend des Mc Burneyschen Punkts resp. das Hypogastrium. Nierengegend unempfindlich. Keine Darmperistaltik sichtbar. Genitalbefund ohne Besonderheiten.

Eine Diagnose war mit Sicherheit nicht zu stellen weder für Perityphlitis, noch für eine Gallensteinerkrankung waren genügende Anhaltspunkte vorhanden. Als bloße Begleiterscheinung der aus dem obigen Befunde sich ergebenden Pneumonie konnte der starke Meteorismus andererseits anch nicht wohl angesehen werden.

Die Frau erholte sich in den nächsten Tagen schnell, die Temperatur kehrte in 3 Tagen zur Norm zurück, auf Glyzerineinläufe erfolgten reichliche Darmentleerungen von normaler Farbe und Konsistenz. Der Bauch wurde weicher, doch war auch weiterhin nichts Bestimmtes durchfühlbar. Die pneumonischen Erscheinungen änderten sich nur insofern, als das Bronchialatmen verschwand; das Knisterrasseln blieb bestehen und ging nur vereinzelt in deutliche Lösungsgeräusche über. Da kein Sputum ausgeworfen wurde, konnte über die Natur der Infiltration nichts ausgesagt werden. Immerhin konnte es sich nicht um eine einfache kruppöse Pneumonie handeln, da kein Schüttelfrost, kein Herpes und überhaupt kein akuter schwerer Beginn vorangegangen waren. Endlich war die mangelhafte Lösung bei Fieberfreiheit absonderlich.

Am 30. IV. ${ }^{3 / 4} 6$ Uhr morgens wird die Pat. auf die Badebank gelegt, um gewaschen zu werden. Die Stationsschwester wendet sich einen Augenblick ab, als die Frau ruft: „Mir wird so schlecht!" Im selben Moment fällt sie zurück, verfärbt sich und hat keine Luft. Die Schwester lảßt mich sofort holen. Es vergehen mindestens 2-3 Minuten bis zu meiner Anwesenheit. Beim ersten Anblick der Frau steht mir die Diagnose Lungenembolie mit voraussichtlich tödlichem Ausgang fest. Nur 
um das klinische Bild zu vervollständigen, wird noch eine ganz kurze Untersuchung vorgenommen, denn höchste Eile tut not:

Fahle Blässe mit nur leicht cyanotischer Tönung überzieht die kähle teuchte Haut; Ausdruck höchster Angst prägt sich in den Gesichtszügen, tiefe geräuschlose Atmung, die in der Exspiration jedesmal von monotonem Stöhnen begleitet ist, unter Ausnutzung der Hilfsmuskeln; weite reaktionslose Pupillen; Puls kaum fühlbar; über Lungen und Herz vorne keine abnormen Geräusche hörbar.

Die Frau wird in aller Eile in den Operationssaal gebracht, auf den Tisch gelegt und eine oberflachliche Chloroformnarkose eingeleitet. Der Zustand verschlechtert sich von Minute zu Minute. Derweil sind die nötigen Instrumente, die - wie ein Tracheotomiebesteck - immer fertig sterilisiert parat gehalten werden, bereit gelegt und die notwendigste Assistenz zur Stelle. Darüher sind wohl noch wieder ca. 10 Minuten vergangen, so daß etwa 20 Minuten nach Eintritt der Embolie mit der Operation begonnen werden kann.

Bogenfürmiger -Hautmuskelschnitt mit sternaler Basis, einem horizontalen Schenkel im 1. Intercostalraum, einem senkrechten 8-10 cm vom linken Sternalrand entfernten und einem zweiten horizontalen im 3. Intercostalraum. Zurückklappen des Lappens auf das Sternum nach Ablösung der Muskeln von den Rippen. Bei den ersten Hautschnitten äußert die Patientin noch Schmerz, dann wird sie still. Schräge Durchschneidung der Knorpel der 2. and 3. Rippe in der Richtung von vorne median nach hinten lateral in der Nähe der Knorpelknochengrenze so, daß die Mammaria int. gerade geschont wird. - Nach Durchtrennung der Weichteile des 1.--3. Zwischenrippenraums und breiter Eröffnung der Pleura wird mit dem Elevatorium der Rippenlappen herausgehebelt und unter Zuhilfenalıme von Intercostalschnitten im 1. und 3. Intercostalraum der Lappen nach außen gebogen. Dabei werden die Rippen etwa dem vertikalen Hautschnitt entsprechend frakturiert.

Die linke Lunge kollabierte, die Herztätigkeit beschränkte sich - soweit das in der Eile beobachtet werden konnte - auf ganz vereinzelte träge Zusammenziehungen. Seine Lage erschien gleich auffallend hoch. Unter gewöhnlichen Verhältnissen präsentiert sich nach Eröffnung der 2. und 3. Rippe das obere Ende des Perikards mit den großen Gefäßen, in diesem Fall lag der größere Teil des Herzens selbst der Thoraxwunde gegenüber, während sich Aorta und Pulmonalis hinter den oberen Wundrand zum Teil verbarg.

Es folgte nun die Eröffnung des oberen Perikards zwischen 2 Pinzetten, was bei der mangelhaften Herzaktion und der nicht so enorm prallen Füllung des r. Herzens ohne Schwierigkeiten vonstatten ging. Es zeigte sich aber gleich, daß die Manipulationen 
Ein Fall v. Embolie d. Lungenarterie n. d. Meth, von Trendelenburg oper, 285

an der Pulmonalis durch ihre abnorme Lage erschwert sein würden, deswegen wurde nachträglich noch ein Stück der 4. Rippe reseziert.

Die weiteren Maßnahmen ließen sich fast genau nach den von Herrn Geheimrat Trendelenburg gegebenen Vorschriften ausführen: fast spielend gelingt die Umgehung der beiden großen Gefäße mit der für die Umlegung des Schlauches bestimmten Sonde, die unter leicht schraubender Bewegung durch den Sinus pericardii transversus durchgeschoben wird. Sobald das olivenförmige Ende unter dem 1. Sternalrand vor den Gefäßen wieder zum Vorschein kommt, Anschraubung des Gummischlauches, wobei ein Assistent das andere Schlauchende in der Richtung des Schraubengewindes mitdreht. Die Sonde wird zurückgeführt und von dem Schlauch durch Scherenschnitt getrennt. Der Schlauch liegt hinter Aorta und Pulmonalis zur Kompression bereit. Alles dieses läßt sich in wenigen Augenblicken bewerkstelligen.

Jetzt galt es, keine Zeit mehr zu verlieren, denn die Patientin war absolut reaktionslos, lag wie tot da, die A tembewegungen hatten aufgehört. Ich verzichtete daher auf die in der Trendelenburgschen Vorschrift erwähnte Ablösung des viszeralen Perikards von der Pulmonalis, die wieder eine gewisse Zeit in Anspruch genommen hätte zumal bei der wiederholt erwähnten Hochlagerung des Gefäßes und incidierte direkt etwas oberhalb des Klappenrings und an der Außenseite das Gefäß in der Längsrichtung, während der Gummischlauch unter Kreuzung der Enden angezogen wurde. Es entstand eine etwa $1 \mathrm{~cm}$ lange Wunde, die genügte, um die zur Extraktion bestimmte Polypenzange in das Lumen einzuführen. Im linken Hauptast fühlte man sofort zwischen den zufassenden Branchen des Instruments einen weichen elastischen Widerstand. Der vorsichtig zurückgezogenen Zange folgt, mit dem einen Ende, wie ein Wurm im Schnabel eines Vogels eingeklemmt, ein großer Embolus. Kaum ist er ganz herausgezogen - wobei ich keinerlei Widerstand oder Einreißen fühlte - so drängt sich schon ein zweiter aus der Gefäßwunde heraus und zwar wird ihm keine Zeit gelassen, gestreckt das Gefäßrohr zu verlassen, sondern neben dem vorderen Ende quillt gleich ein hinteres Stück in Windungen hervor, so daß es fast zu einer Einklemmung kommt. Die Herausbeförderung der beiden großen Emboli erfolgt auf diese Weise in wenigen Sekunden.

Es folgt die wandständige Abklemmung der Gefäßwunde mit der gebogenen Klemmzange unter Anheben der Wunde mit der mit nach außenstehenden Häkchen versehenen federnden Pinzette. So- 
fort wird die Kompression weggelassen, das Gefäßrohr füllt sich prall. Ein Blutverlust ist nicht erfolgt.

Unmittelbar an das Hervorkommen des zweiten Embolus schließt sich ein tiefer A temzug. Die Herztätigkeit sistiert aber vollständig und kehrt nur allmählich wieder unter rhythmischen mechanischen Reizen, die jetzt nach provisorischer Abklemmung der Pulmonaliswunde in aller Ruhe ausgeführt werden können. Erst nachdem wieder kräftige Aktion unter regelmäßiger Folge von Vorhöfen und Kammern eingesetzt hat, die Atmung unter Sauerstoffzuführung gleichmäßig und ruhig geworden ist, wird zur Naht der Gefäßwunde geschritten.

Da hatte ich nun einige Schwierigkeiten, vor allem bedingt durch die unbequeme Lage der Pulmonalis. Gleich zu Beginn zeigte es sich, daß die Wundränder sich langsam durch die Klemme zurückzogen, was einerseits durch die pralle Füllung der Pulmonalis vor allem aber dadurch hervorgerufen schien, daß an der Klemme zur Erleichterung der Naht ein Zug nach unten ausgeübt werden mußte. Dadurch wurde das durch den nach hinten gerichteten Verlauf der Pulmonalis an und für sich schon stärker angezogene periphere Wundende unter zu hohe Spannung versetzt und rutschte zwischen die Branchen der Klemme zurück. Unter erneuter kurz dauernder Kompression mit dem Schlauch mußte die Wunde ein parmal neu gefaßt werden. Schließlich gelang aber der vollkommene Verschluß des kräftig pulsierenden Gefäßes. Zwischen den Seidenknopfnähten quoll nicht ein Tropfen heraus.

Derweil wurde der Radialpuls wieder fühlbar, während das Aussehen der Patientin noch blaß und cyanotisch blieb. - Die Narkose war seit Eröffnung des Thorax ausgesetzt worden, da sie nicht mehr notwendig war.

Mittelst dreier Katgutnähte wurde das Perikard übernäht und dann der Rippenlappen in seine Lage gebracht, durch einige Periostund Muskelnähte fixiert. Der luftdichte Verschluß der Pleura erfolgt durch die Naht des Pectoralis. Hautnaht mit Dränage.

Das BewuBtsein ist jetzt wiedergekehrt, auch das Aussehen ist frischer geworden, der Puls besser gefüllt. Die Pat. stöhnt über Schmerzen in der Herzgegend. Die Pupillen reagieren. Extremitäten und Gesicht noch kühl. Unter weiterer Sauerstoffzufuhr, wie sie Pat. anfänglich als angenehm empfindet, später ablehnt, künstlicher Erwärmung erholt sie sich zunehmend: die Cyanose läßt nach, die Lippen färben sich blaßrot, die Glieder nehmen natürliche Wảrme an und unter $1 \mathrm{cg}$ Morph. tritt ruhiger Schlaf ein. Um 9 Uhr ist Pat. wieder wach, aber ruhiger, die Schmerzen 
Ein Fall v. Embolie d. Lungenarterie n. d. Meth. von Trendelenburg oper. 287

sind mäßiger, so daß sie spontan nicht mehr klagt. Puls 110, weich, aber ganz gut gefüllt, Atmung tief, 42 p. min.

Im Laufe des Tages ist der Zustand wechselnd ein besserer und ein schlechterer. Es wirl 1/2 stündlich Kampfer gegeben, um die Herztätigkeit anzuregen, daneben Kochsalzeinläufe und per os schluckweise Tee und Kognak.

Akute Verschlimmerungen; die auf Blutung oder erneute Embolie schließen ließen, treten nicht ein, nur hält eine mäßige Cyanose dauernd vor und die Atmung bleibt frequent. Pulsfrequenz schwankt zwischen 100 und 120.

Besondere Geräusche sind weder über dem Herzen noch über der linken Lunge zu hören. 'Deutliche reine, etwas dumpfe Herztöne bei regelmäßiger Aktion, reines Vesikuläratmen über der linken Lange bald wieder hörbar, das ein Weiterbestehen des Pneumothorax unwahrscheinlich macht.

Von 7 Uhr abends ab verschlechtert sich der Zustand deutlich. Die Gesichtszüge fallen ein, die Nase wird spitz, die Extremitaten kühlen ab, die Axillartemperatur sinkt unter $35^{\circ} \mathrm{C}$. Das BewuBtsein bleibt erhalten. 91/4 Uhr abends nach kurzer motorischer Unruhe unter lautem Schreien plötzlicher Exitus.

Die Sektion wurde von den Angehörigen abgelehnt, wir muBten uns laher mit einer notdürftigen Inspektion von der Wunde aus begnügen, die immerhin über das betreffs der Operation Wissenswerte einigermaßen ausreichenden AufschluB gab: die Lungen hatten sich ausgedehnt, der Pneumothorax war behoben, das Herz lag, rom Perikard bedeckt, an normaler Stelle, abgesehen von der auch jetzt bestätigten Hochdrängung verbunden mit Hochstand der Zwerchfellkuppe. In der Pleura findet sich nur eine geringe Menge blutiger Flüssigkeit, der Herzbeutel ist leer, keinerlei Koagula. Das Herz ist schlaff und erweitert. Die Naht der Pulmonalwunde hat gehalten. Im Herzen ein wenig umfangreiches Speckgerinnsel. Der Hauptstamm der Pulmonalis ist leer. Man sieht auf der Innenfläche des Gefäßrohrs die glatte, etwas blutig tingierte Naht und gegenüber eine feine lineäre Verletzung der Intima, sonst keinerlei Schàdigung derselben. Mit dem Finger fühlt man in den Ästen der Pulmonalarterie ebenfalls keinerlei Thromben und es gelingt auch nicht, mit der Kornzange aus tieferen Ästen Gerinnsel zu extrahieren, nur aus einem Ast 2. oder 3. Ordnung der rechten Pulmonalis werlen kleine, ziemlich festhaftende Thrombusstücke herausgeholt.

Was beweist der geschilderte Fall, stellt ex einen wesentlichen Fortschritt dar, welche Lehren müssen wir aus ihm ziehen?

Die schweren Erscheinungen, unter denen die Embolie einsetzt, machen von vorne herein, eine totale oder wenigstens nahezu totale Verlegung beider Hauptäste der Pulmonalis, demnach den tödlichen Ausgang des Anfalls so gut wie sicher. Die rapide Verschlechterung des Zustandes bestätigt die Annahme. Da gelingt es nun. auf operativem Wege das Zirkulationshindernis, die beiden großen Emboli zu beseitigen und die Frau noch 15 Stunden am Leben zu erhalten. Diese nackte Tatsache bedeutet gewiß einen bedeutenden 
Fortschritt auf dem eingeschlagenen Wege, einen erfreulichen Erfolg der Trendelenburgschen Operation, deren Durchführbarkeit am Menschen damit bewiesen ist.

Oder sollte etwa der doch erfolgte Exitus diese Schlüsse als verfrüht und unberechtigt erscheinen lassen? Wir glauben nicht. Es kann als erwiesen angesehen werden, daß der 'Tod nicht der Operation zuzuschreiben ist, so daß man also nicht sagen kann, es werde für eine Todesursache eine andere eingesetzt. Die Autopsie zeigt, es sind wieder normale Verhältnisse hergestellt, nur das Herz ist zu schwach gewesen, den schweren Insult zu überwinden.

Warum das Herz sich nicht wieder dauernd erholte, läßt sich nicht mit Sicherheit sagen, dazu ist der Fall ja leider nicht genügend klargestellt. Doch darf man wohl annehmen, daß das Herz durch das Grundleiden in Verbindung mit dem Meteorismus bereits geschwächt war, wofür die hohe Frequenz des etwas weichen Pulses bei der Aufnahme sprach, daß vermutlich schon die Pneumonie des r. Unterlappens nichts anderes gewesen ist als ein embolischer Infarkt - der Verlauf, der Sitz, das eigentümliche anhaltende Knistern (vgl. Eichhorst, Deutsche med. Wochenschr., Nr. 15, 1908) sprechen dafür. Dem allen folgt der schwere embolische Insult, der zur Zeit der Operation schon zu völligem Versagen der Herztätigkeit geführt hat. Nun ist nicht zu leugnen, daß auch die verschiedenen Manipulationen der Operation natürlich noch neue Angriffe auf die Leistungsfähigkeit des Herzens darstellen und daß vielleicht doch die Verlängerung der Operation durch das Abrutschen der Seitenklemme und das dadurch wiederholt erforderliche Abklemmen der großen Gefäße schädlich gewirkt hat. Es wird aber sicher Fälle geben, wo das Herz - besonders bei etwas langsamerem Verlauf der embolischen Erscheinungen und dem dadurch ermöglichten relativ früheren Eingreifen noch leistıngsfähig genug ist, die Operation dauernd zu überstehen.

Eine Schädigung des Zentralnervensystems von längerer Dauer wird durch die Gefäßabklemmung nicht gesetzt, das beweist das alsbald zurückkehrende Bewußtsein, das völlig normale Verhalten der empfindlichen Pupillenreflexe und überhaupt die Wiederaufnahme aller physischen und psychischen Funktionen. Die Gefäßnaht war dicht und wird in der späteren Zeit der Heilung ja kaum heftigeren Druckschwankungen ausgesetzt, als zu Anfang nach Wiederkehr des Kreislaufs. Die kleine Verletzung der Intima, die vielleicht der Incision zuzuschreiben ist, würde voraussichtlich binnen kurzem endothelisiert sein und würde sich in einem anderen 
Ein Fall v. Embolie d. Lungenarterie n. d. Meth. von Trendelenburg oper. 289

Falle auch sicher vermeiden lassen. Die Gefahr der Infektion kann nicht größer sein als bei einem Fall von Herznaht bei einer Schuß-oder Stichwunde des Herzens.

Der erwähnten Schwierigkeit der Seitenabklemmung muß Rechnung getragen werden, indem für solche Fälle Klemmen festerer Konstruktion und etwas veränderter Krümmung vorrätig sein müssen. Im übrigen hat sich das von Herrn Geheimrat Trendelenburg konstruierte Instrumentarium vollständig bewährt und bedarf keinerlei Hinzufügung oder Änderung auf Grund des neuen Falls. Auch der Gang der Operation bis in alle Einzelheiten hat sich als durchweg zweckmäßig und leicht ausführbar erwiesen.

Besonders hervorzuheben ist die große Schnelligkeit, mit der sich die Emboli aus der geöffneten Pulmonalis extrahieren ließen. Es hat sich gezeigt, daß die prall gefüllte Arterie infolge ihrer großen Dehnbarkeit und Elastizität die Emboli von selbst heraus zu drücken bemüht ist und dem Operateur so zu Hilfe kommt. Die Kompression der großen Gefäße braucht also nur für ganz kurze Zeit angewandt zu werden und braucht bei dem Darniederliegen der Herzaktion wahrscheinlich auch gar keine ganz vollständige zul sein.

Indes können mit der Technik allein nicht die Erfolge garantiert werden. Eine wesentliche Hauptsache ist natürlich die Sicherheit der Diagnose und die sofortige Erkennung der Prognose des Krankheitsbildes. Die Symptomatik in diesem Falle war einfach und klar, und sie wird es meist sein in den Fällen, wo überhaupt an die Operation gedacht werden soll, in den Fällen von totalem oder nahezu totalem Verschluß der Pulmonalis.

Die Chancen der Lungenembolieoperation stehen und fallen aber mit der Möglichkeit des sofortigen Eingriffs. Um diese Bedingung zu erfüllen, sind unbedingt folgende Anordnungen erforderlich:

1. es muß immer ein Arzt zur Verfügung stehen, welcher sich durch Übungen an der Leiche oder vielleicht auch am lebenden Tiere auf die Operation einge übt hat;

‥ auf jeder Station muß die vorstehende Schwester oder Oberpflegerin auf die Symptome der Lungenembolie hingewiesen und über ihre Operationsmöglichkeit trotz der Schwere der Erscheinnngen aufgeklärt sein und die Anweisung haben, ohne jeden Verzug den Arzt zu benachrichtigen; endlich 
3. muß das Instrumentarium stets aseptisch und vollständig bereit gehalten werden.

Zum Schluß möchte ich noch darauf hinweisen, daß, wie der Verlauf des Falls gezeigt hat, eine Ausschaltung des Pneumothorax

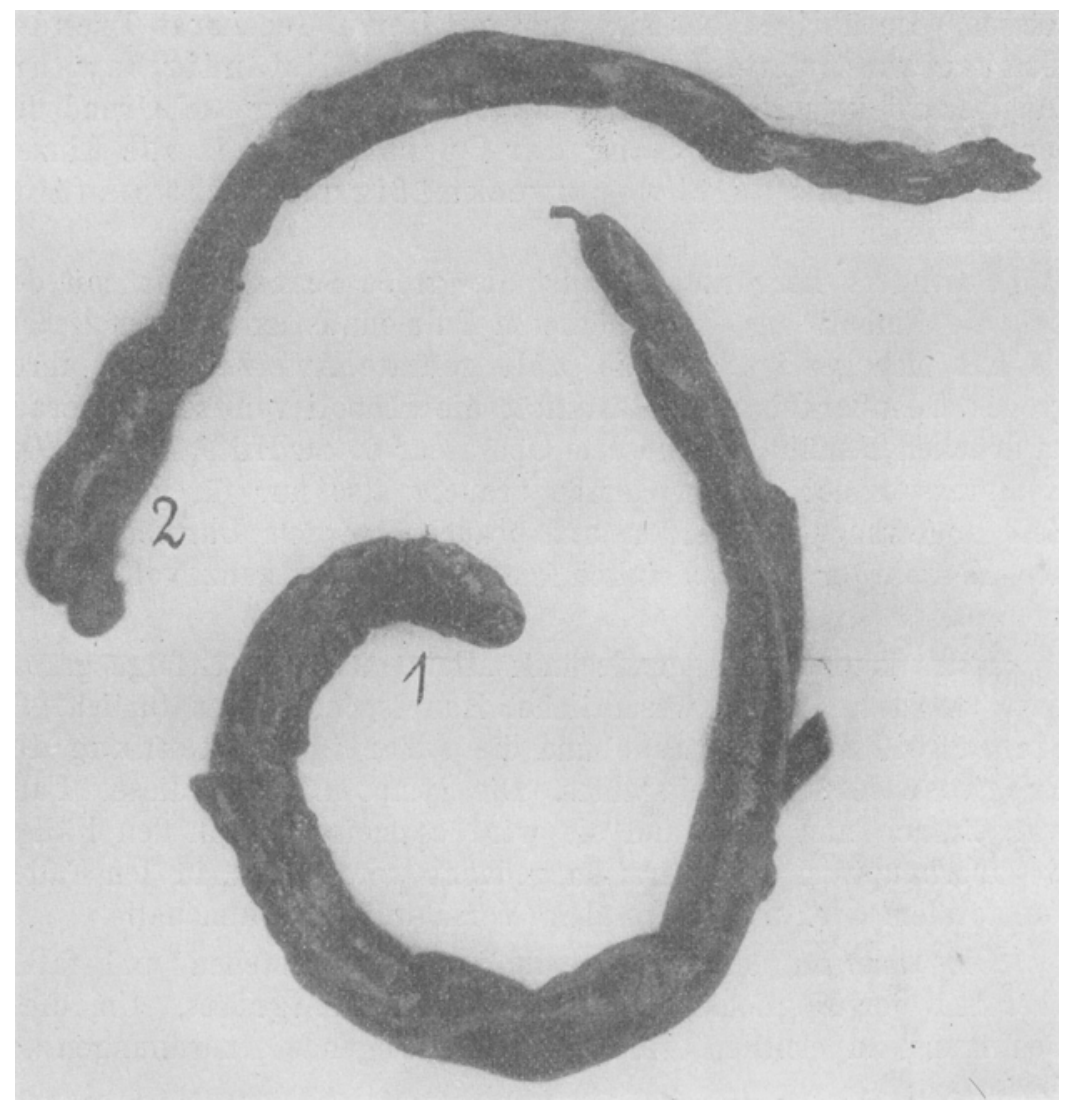

Die beiden extrahierten Emboli. (Naturliche Große.)

bei der Operation durch die Sanerbruchsche Kammer oder den Brauerschen Apparat nicht als conditio sine qua non für die Operation angesehen werden kann, da der Pneumothorax bei genügendem Wundverschluß in kurzer Zeit verschwindet und die Lungen wieder ausgedehnt werden. Ist genügend Zeit und Assistenz zur Verfügung, so würden wir gewiß nicht anstehen. den Brauer-Drägerschen 
Ein Fall v. Embolie d. Lungenarterie n. d. Meth. von Trendelenburg oper. 291 Apparat zu benutzen und damit vielleicht noch etwas schonender verfahren.

Der zuerst extrahierte Embolus (1) hat eine Länge von $21 \mathrm{~cm}$, einen Durchmesser von $10-12 \mathrm{~mm}$; am Ende verjüngt er sich etwas. Der andere (2) mißt $15 \mathrm{~cm}$ in der Länge, $7-9 \mathrm{~mm}$ im Durchmesser, auch er nimmt allmählich an Umfang ab. Schwarze Blutniederschläge verdecken zum großen Teil die eigentliche hellerrote Färbung, sowie die bald körnige, bald mehr gerippte Oberflächenzeichnung. Aus der Form muß man schließen, daß es sich umı zwei nicht zusammengehörige Thromben handelt.

Die nach dem Tode in einem Aste der rechten Pulmonalis noch vorgefundenen festhaftenden kleinen Thromben (vergl. S. 287) sind vermutlich die Urheber des vorangegangenen embolischen Infarkts. 